\title{
Actions of Superrigid Non-Kazhdan Lattices on Compact Manifolds
}

\author{
EDWARD R. GOETZE
}

University of Michigan, 317 West Engineering, Department of Mathematics, Ann Arbor, MI 48109, U.S.A.

(Received: 23 July 1993)

\begin{abstract}
In this paper, we examine the actions of lattices in superrigid non-Kazhdan simple groups on compact manifolds. The geometric results are obtained by analyzing the properties of amenable ergodic groupoids.
\end{abstract}

Mathematics Subject Classifications (1991): 22E40, 28D05, 58D40.

In this paper, we study the action of a discrete Lie group on a compact manifold, continuing the investigation begun by Zimmer in [6]-[8]. In particular, in [8], Zimmer was able to deduce a number of geometric consequences by using Kazhdan's property as a replacement for measurable superrigidity for cocycles. This was done, in part, by analyzing the algebraic hull of a cocycle from a Kazhdan group into an amenable group. Since many Kazhdan groups are superrigid, i.e. satisfy the hypotheses of measurable superrigidity [5, Ch. 5], one would hope that similar results hold true in the case of superrigid non-Kazhdan groups. We establish such results in this paper.

THEOREM 1. Let $\Gamma$ be an irreducible lattice in $G$ a semisimple Lie group with finite center, $\mathbb{R}$-rank $(G) \geq 2$, and no compact factors. Suppose $\Gamma$ acts ergodically on a compact manifold $M$ of dimension 2 preserving a volume density and a connection. Then $M=S^{2}$ or $P_{2}(\mathbb{R})$.

THEOREM 2. Let $\Gamma$ and $G$ be as in Theorem 1. Suppose $\Gamma$ acts ergodically on a compact manifold $M$ preserving a Lorentz metric. Then either,

(1) $\Gamma$ leaves a Riemannian metric invariant and hence the action factors to $\Gamma \rightarrow K \rightarrow \operatorname{Diff}(M)$ where $K$ is a compact Lie group, or

(2) $\Gamma$ factors to $\Gamma \rightarrow \operatorname{SL}(2, \mathbb{R}) \times K \rightarrow \operatorname{Diff}(M)$ where $K$ is a compact Lie group. In this case, the simple components of $G$ must all be locally isomorphic to $\mathrm{SL}(2, \mathbb{R})$.

These results are generalizations of theorems by Zimmer in [6], [8], where $\Gamma$ was assumed to be a Kazhdan group. Consequently, the proofs of the theorems here will assume $\Gamma$ to be a non-Kazhdan group, i.e. a lattice in a product of rank 1 simple groups. 
We assume the reader is familiar with the elements of the theory of superrigidity for cocycles as presented in [5] and [9].

\section{Amenable Ergodic Groupoids}

Before beginning the actual proof of these theorems, it is necessary to discuss some related topics. First, we wish to show that the algebraic hull of the cocycle of a class of lattices cannot be amenable. To do this we will consider the properties of ergodic groupoids.

Let $S$ be an ergodic $G$ space. Then $S \times G$ naturally defines an ergodic groupoid $\mathcal{G}$. Let $R(\mathcal{G})$ be the naturally associated principal ergodic groupoid, i.e.

$$
R(\mathcal{G})=\{(s, t) \mid \exists g \ni s . g=t\} \subset S \times S .
$$

Next, define $S(\mathcal{G})=\left\{(s, g) \mid g \in G_{s}\right\} \subset S \times G$. Note that there exists natural maps $i: S(\mathcal{G}) \rightarrow \mathcal{G}$ and $p: \mathcal{G} \rightarrow R(\mathcal{G})$ defined by $i(s, g)=(s, g)$ and $p(s, g)=(s, s . g)$. As in [4], we have an exact sequence of ergodic groupoids

$$
0 \rightarrow S(\mathcal{G}) \rightarrow \mathcal{G} \rightarrow R(\mathcal{G}) \rightarrow 0
$$

Recall the definition for an ergodic groupoid to be amenable: $\mathcal{G}$, a measured groupoid, is amenable if for every separable Banach space $\mathrm{E}$, and for every cocycle $\phi: \mathcal{G} \rightarrow \operatorname{Iso}(E)$, the set of isometric automorphisms of $E$, and for every measurable field $\mathcal{U}_{\mathcal{G}} \rightarrow E_{1}^{*}$ invariant under $\phi\left(E_{1}^{*}\right.$ is the unit ball in $E^{*}, \mathcal{U}_{\mathcal{G}}$ is the units in $\mathcal{G}$, a measurable field is an assignment for every $x \in \mathcal{U}_{\mathcal{G}}, A_{x} \subset E_{1}^{*}$ a compact convex subset such that $\left\{\left(x, A_{x}\right)\right\} \subset S \times E_{1}^{*}$ is measurable, and invariant meaning $\phi^{*}(x) A_{d(x)}=A_{r(x)}, d(x)$ the right identity of $x$, and $r(x)$ the left identity of $x$ ), then there exists a fixed point in $F\left(\mathcal{U}_{\mathcal{G}},\left\{A_{x}\right\}\right)$, i.e. there exists a function $a: \mathcal{U}_{\mathcal{G}} \rightarrow E^{*}$ such that $a(x) \in A_{x}$ and $\phi^{*}(x) a(d(x))=a(r(x))$.

LEMMA 3. If $S(\mathcal{G})$ and $R(\mathcal{G})$ are amenable, then so is $\mathcal{G}$.

Proof. Let $\phi$ and $\left\{\left(x, A_{x}\right)\right\}$ be as above. Since $S(\mathcal{G})$ is amenable, the set of $S(\mathcal{G})$ fixed points in $F\left(\mathcal{U}_{\mathcal{G}},\left\{A_{x}\right\}\right)$ is nonempty. Call this set $A_{0}$. Thus $a \in A_{0}$ means $\phi^{*}(s, g) a(d(s, g))=a(r(s, g))$ where $g \in G_{s}$. But we have that $d(s, g)=$ $(s . g, e)$ and $r(s, g)=(s, e)$. Since $g \in G_{s}$, this translates to $\phi^{*}(s, g) a(s)=a(s)$ (viewing $a: S \rightarrow A_{x}$, since $S \cong \mathcal{U}_{\mathcal{G}}$ ). For the moment, assume that $A_{0}$ is a $\mathcal{G}$ invariant set in $F\left(S,\left\{A_{s}\right\}\right)$. Since $S(\mathcal{G})$ fixes $A_{0}$, we have a natural action by $R(\mathcal{G})$ on $A_{0} \subset F\left(S,\left\{A_{s}\right\}\right)$ (note that $\left.\mathcal{U}_{R(\mathcal{G})} \cong S\right)$. Since $R(\mathcal{G})$ is amenable, there exists an $R(\mathcal{G})$ fixed point in $a_{0}$, we must then be a $\mathcal{G}$ fixed point. Therefore $\mathcal{G}$ is amenable. 
Thus, it remains to show that $A_{0}$ is a $\mathcal{G}$ invariant set. We need to show that $g . a \in A_{0}$ provided $a \in A_{0}$. Since $(g . a)(s)=\alpha^{*}(s, g) a(s, g)$, this amounts to showing that $\left(g_{s} \cdot(g . a)\right)(s)=(g \cdot a)(s)$ where $g_{s} \in G_{s}$. But we have

$$
\begin{aligned}
\left(g_{s}(g . a)\right)(s) & =\alpha^{*}\left(s, g_{s}\right) \alpha^{*}(s, g) \phi\left(s g_{s} g\right) \\
& =\alpha^{*}\left(s, g_{s} g\right) \phi\left(s g_{s} g\right) \quad\left(\text { since } g_{s} \in G_{s}\right) \\
& =\alpha^{*}\left(s, g g_{s g}\right) \phi\left(s g g_{s g}\right) \quad\left(\text { where } g_{s g} \in G_{s g}\right) \\
& =\alpha^{*}(s, g)\left(\alpha^{*}\left(s g, g_{s g}\right) \phi(s g)\right) \\
& =\alpha^{*}(s, g) \phi(s g)=(g . a)(s),
\end{aligned}
$$

thus completing the proof.

We will now make use of Lemma 3 to prove the following.

THEOREM 4. Suppose $S$ is an ergodic $G$-space, and $\alpha: S \times G \rightarrow H$ is a cocycle. Suppose also that $G$ acts tamely on $S \times_{\alpha} H$. If $H$ is amenable and all stabilizers of $G$ on $S$ are amenable, then $G$ acts amenably on $S$.

Proof. Note that there exists a stable orbit equivalence between the $G$ action on $S$ and the $H$ action on $\left(S \times_{\alpha} H\right) / G$. This follows simply because there is a 1-1 correspondence between the $G$ orbits on $S$ and the $H$ orbits on $\left(S \times_{\alpha} H\right) / G$. If $G$ acts tamely on $S \times{ }_{\alpha} H$ then the Mackey range of the $G$ action on $S$ is the $H$ action on $\left(S \times_{\alpha} H\right) / G$. This provides a stable orbit equivalence between the $H$ action on the Mackey range and $R(\mathcal{G})$, which is just the groupoid formed from the $G$ orbits on $S$. Since stable orbit equivalence preserves amenability, the amenability of $H$ implies that $R(\mathcal{G})$ is amenable. Since all the stabilizers of the $G$ action on $S$ are amenable, we have that $S(\mathcal{G})$ is also amenable. The result then follows immediately from Lemma 3.

We now provide one more result that will be useful in the proof of Theorem 1 .

PROPOSITION 5. Let $\Gamma \subset G_{\mathbb{R}}^{0}$ be an irreducible noncocompact lattice where $\mathbb{R}-\operatorname{rank}(G) \geq 2$. If $\phi: \Gamma \rightarrow K$ is a homomorphism where $K$ is a compact Lie group, then $\phi$ has finite image.

Proof. We may assume that $\overline{\phi(\Gamma)}=K$. Since $\Gamma$ is F-simple, it suffices to show that the kernel of $\phi$ is infinite. If $G$ has a nontrivial center, we obtain a map

$$
\Phi: \Gamma /(Z(G) \cap \Gamma) \rightarrow K / \phi(Z(G) \cap \Gamma) .
$$

Since $Z(G) \cap \Gamma$ is finite and normal, and as $\overline{\phi(\Gamma)}=K, \phi(Z(G) \cap \Gamma)$ is also finite and normal. Hence if $\Phi$ has finite image, so does $\phi$. So we may reduce to the case where $G$ has trivial center. By [5, Th. 6.1.10], we may assume that $\Gamma$ is the $\mathbb{Z}$-points of a semisimple $\mathbb{Q}$-group $H$, and if $K=L_{\mathbb{R}}$ for some $\mathbb{R}$-group $L$, then $\phi: H \rightarrow L$ is an $\mathbb{R}$-map. Since $\Gamma$ is a noncocompact lattice, by $[5,6.1 .9], H_{\mathbb{Q}}$ has nontrivial unipotent elements, and hence so must $H_{\mathbb{Z}}$. In fact, $H_{\mathbb{Z}}$ must then contain an infinite 
number of nontrivial unipotents. Since the image of a unipotent element under $\phi$ must again be a unipotent, and as $K$ is compact, we have that all the unipotents in $H_{\mathbb{Z}}$ are in the kernel of $\phi$. Thus the kernel is infinite.

\section{Proof of Theorem 1}

Since $\Gamma$ acts ergodically, and finite actions cannot be ergodic on manifolds of positive dimension, to demonstrate the action is trivial it suffices to see that it is finite. Let $\alpha: M \times \Gamma \rightarrow \operatorname{SL}(2, \mathbb{R})$ be the cocycle determined by the natural induced action of $\Gamma$ on $P(M)$, the frame bundle over $M$. Since the $\Gamma$ action preserves a volume density, we may safely assume that the image of $\alpha$ does lie inside $\operatorname{SL}(2, \mathbb{R})$. Let $H$ be the algebraic hull of the cocycle $\alpha$. If $H$ is compact (i.e. $H=\mathrm{O}(2, \mathbb{R}))$, the $\Gamma$ action on $M$ must preserve a measurable Riemannian metric on $M,[9$, Prop. 2.2]. In addition, $\Gamma$ preserves a connection, so by [9, Th. 2.5], $\Gamma$ preserves a smooth Riemannian metric on $M$. Hence, there exists a homomorphism $\Theta: \Gamma \rightarrow \operatorname{Isom}(M)$. As $M$ is compact and 2-dimensional, $\operatorname{Isom}(M)$ is compact and at most 3-dimensional. Thus $\overline{\Theta(\Gamma)}$ is either abelian or $\mathrm{O}(3, \mathbb{R})$ (or $\mathrm{SO}(3, \mathbb{R})$ ). In the former, since $\Gamma$ is F-simple, $\Theta(\Gamma)$ must be finite. In the latter case, [2, Th. 2.3.1] implies that $M$ must be either $S^{2}$ or $P_{2}(\mathbb{R}), 2$-dimensional projective space. Note that Proposition 5 assures us that this cannot happen in the noncocompact case.

So, we may assume that $H$ is noncompact. Since $\Gamma$ acts preserving a connection, we have a homomorphism $\phi: \Gamma \rightarrow A(M)$, the affine transformations on $M$. Since there is a natural inclusion of $A(M)$ in Isom $(P(M))$, we have a homomorphism

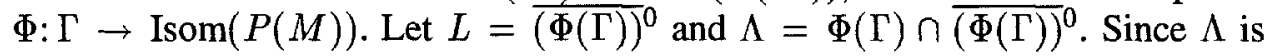
normal in $\Phi(\Gamma)$, using the F-simplicity of $\Gamma$, we have $\Lambda$ is either finite or of finite index in $\Phi(\Gamma)$. So, we may assume that $\Lambda$ is either trivial or $\Phi(\Gamma)$.

If $\Lambda$ is trivial, then $\Phi(\Gamma)$ is closed in $\operatorname{Isom}(P(M))$, and therefore $\Gamma$ acts properly on $P(M)$. Discreteness of $\Gamma$ then implies that $\Gamma$ acts tamely with finite stabilizers. Since $H \subset \operatorname{SL}(2, \mathbb{R})$ and is noncompact, $H$ is either $\operatorname{SL}(2, \mathbb{R})$ or is amenable. If $H$ is SL(2), applying Superrigidity we have that $\alpha$ is equivalent to a cocycle corresponding to a homomorphism $\beta: \Gamma \rightarrow \mathrm{SL}(2)$, which extends to a homomorphism $\beta: G \rightarrow \mathrm{SL}(2)$. This is equivalent to the existence of a measurable trivialization of $P(M)$ to $M \times \operatorname{SL}(2)$ such that $\Gamma$ acts by the product action (where $\Gamma$ acts on SL(2) via $\beta$ ). By [1], the existence of such a $\beta$ is impossible unless the Lie algebra of $G$ consists of simple Lie algebras all of whose complexifications equal $\operatorname{sl}(2, \mathbb{C})$. In this case, irreducibility of $\Gamma$ implies that $\beta(\Gamma)$ is dense in SL(2). Hence, there exists a sequence of elements $\left\{\gamma_{n}\right\} \rightarrow \infty$ in $\Gamma$ such that $\left\{\beta\left(\gamma_{n}\right)\right\} \rightarrow 1$ in SL(2). Select a compact set $K$ containing 1 in SL(2) of nonzero Haar measure. Then for sufficiently large $N, n \geq N$ implies $\gamma_{n} . K \cap K \neq \emptyset$. Hence, $\gamma_{n} .(M \times K) \cap(M \times K) \neq \emptyset$ for sufficiently large $\mathrm{n}$, contradicting the properness assumption.

If $H$ is amenable, noting that the stabilizers are finite and therefore amenable, then applying Theorem 4, $\Gamma$ acts amenably on $M$. However, $M$ is compact and 
therefore of finite volume, so $\Gamma$ itself must be amenable. Since $\Gamma$ is a lattice in $G$, this is clearly impossible.

The final case to consider is that when $\Lambda=\Phi(\Gamma)$, i.e. $\Phi(\Gamma) \subset L$. By [3, Th. 9.6.15], $L$ must be semisimple. Since $H$ is noncompact, $L$ must also be noncompact. ( $L$ compact implies the existence of a smooth invariant Riemannian metric on $M$, which, by [9, Prop. 2.4] implies $\alpha$ is equivalent to a cocycle into $\mathrm{O}(2, \mathbb{R})$. Hence, $H$ is compact.) Thus, we may apply Superrigidity to obtain a homomorphism $G \rightarrow L$. $L$ must therefore contain a group locally isomorphic to a simple component $G^{\prime}$ of $G$, and so must $\bar{\Gamma} \subset A(M)$. By [10, Cor. 3.6], there is an open dense conull set of this subgroup for which the stabilizers are discrete. But this is impossible since any such $G^{\prime}$ is at least 3 dimensional and $M$ is only 2 dimensional.

\section{Proof of Theorem 2}

Retaining the notation from the proof of Theorem 1 it will suffice to show that $L$ must be compact. Again, we have two cases to consider: either $\Lambda$ is trivial or equals $\Phi(\Gamma)$. In the former, we may rule out the possibility that $H$ is amenable (using Theorem 4), hence, we once again obtain a homomorphism $\beta: G \rightarrow H \subset$ $\mathrm{SO}(1, n-1)$. Thus, $H$ must contain a subgroup locally isomorphic to a simple factor of $G$. As in [8, Th. 4.1], this must be locally isomorphic to either $\operatorname{SO}(1, m)$ or $\mathrm{SU}(1, m)$. But, again, irreducibility of $\Gamma$ implies that $\beta(\Gamma)$ is dense in this subgroup. As in Theorem 1, this is impossible.

If $\Lambda=\Phi(\Gamma)$, since $L$ is semisimple and noncompact, we have by Superrigidity a homomorphism $G \rightarrow L$. By $[8$, Th. B], either $L \subset \operatorname{SL}(2, \mathbb{R}) \times K$, where $K$ is a compact Lie group, or $L$ is amenable. F-simplicity of $\Gamma$ rules out the latter possibility, so $L=\operatorname{SL}(2, \mathbb{R}) \times K^{\prime}$. However, this is impossible unless $G$ is of the requisite form (using the main result from [1] and Superrigidity).

\section{References}

1. Johnson, F. E. A.: On the existence of irreducible discrete subgroups in isotypic Lie groups of classical type. Proc. London Math. Soc., 56 (1988), 51-77.

2. Kobayashi, Shoshichi: Transformation Groups in Differential Geometry, Springer-Verlag, New York, 1972.

3. Margulis, G. A.: Discrete Subgroups of Lie Groups, Springer-Verlag, New York, 1991.

4. Caroline Series: An application of groupoid cohomology. Pacific J. Math. 92 (1981), 415-432.

5. Zimmer, R. J.: Ergodic Theory and Semisimple Groups, Birkhauser, Boston, 1984.

6. Zimmer, R. J.: On the automorphism group of a compact Lorentz manifold and other geometric manifolds, Invent. Math. 75 (1984), 425-436.

7. Zimmer, R. J.: Actions of lattices in semisimple groups preserving a $g$-structure of finite type, Ergodic Theory and Dynamical Systems 5 (1985), 301-306.

8. Zimmer, R. J.: Kazhdan groups acting on compact manifolds, Invent. Math. 83 (1986), 411-424.

9. Zimmer, R. J.: Ergodic theory and the automorphism group of a $G$-structure, in C. C. Moore (ed.), Group Representations, Ergodic Theory, Operator Algebras, and Mathematical Physics, Springer, New York, 1987, pp. 247-278.

10. Zimmer, R. J.: Automorphism groups and fundamental groups of geometric manifolds, Proc. Symp. Pure Mathematics, 54 (1993), 693-710. 